\title{
Avaliação da expansão urbana na Cidade de Maceió, Alagoas - Nordeste do Brasil
}

\author{
Evaluation of the urban expansion in the City of Maceió, Alagoas - Northeast of Brazil \\ Evaluación de la expansión urbana en la Ciudad de Maceió, Alagoas - Noreste de Brasil
}

\author{
Bárbara Alves Batista \\ ORCID: https://orcid.org/0000-0002-0034-0991 \\ Universidade Federal de Alagoas, Brasil \\ E-mail: barbara.alba18@gmail.com \\ Washington Luiz Félix Correia Filho \\ ORCID: https://orcid.org/0000-0002-4029-4491 \\ Universidade Federal de Alagoas, Brasil \\ E-mail: wlfcfm@gmail.com \\ José Francisco de Oliveira Júnior \\ ORCID: https://orcid.org/0000-0002-4029-7605 \\ Universidade Federal de Alagoas, Brasil \\ E-mail: jose.junior@icat.ufal.br \\ Dimas de Barros Santiago \\ ORCID: https://orcid.org/0000-0001-7118-8467 \\ Universidade Federal de Campina Grande, Brasil \\ E-mail: dimas.barros91@ gmail.com \\ Carla Taciane dos Santos \\ ORCID: https://orcid.org/0000-0002-6426-9346 \\ Universidade Federal de Alagoas, Brasil \\ E-mail: carlataciane01@gmail.com
}

\begin{abstract}
Resumo
O crescimento das cidades juntamente com a formação desordenada de grandes metrópoles ao redor do mundo resulta em grandes mudanças no uso e ocupação do solo. Porém, há poucos estudos que relacionem a expansão urbana e seus efeitos nas cidades do Nordeste do Brasil (NEB). Assim, o objetivo deste estudo foi avaliar a expansão urbana em Maceió-Alagoas entre 1985 e 2020 a partir de produtos orbitais Land Surface Temperature (LST) e Normalized Difference Vegetation Index (NDVI), com a finalidade de detectar as mudanças e os seus efeitos ambientais. Para isto foram utilizados produtos orbitais adquiridos dos sistemas-sensores Landsat 5/Thematic Mapper (TM) e 8/Operational Land Imager (OLI). No estudo utilizaram-se quatro imagens para a observação da variação espaço-temporal da urbanização, correspondente aos anos de 1987,1998, 2006 e 2020. Os mapas temáticos de NDVI e LST foram gerados a partir do software de ambiente R. Os resultados obtidos apontaram alterações substanciais no uso e ocupação do solo detectado pelo NDVI, e aumento na LST ao longo dos 35 anos. Tal variabilidade ocorreu nos bairros localizados na porção norte e noroeste da cidade, resultante dos programas de incentivo do Governo Federal na década de 2009, principalmente o Complexo do Benedito Bentes (CBB) com as maiores transformações no uso e ocupação do solo, principalmente o maior aumento na LST entre $7,5-10,0^{\circ} \mathrm{C}$. Os efeitos produzidos pela expansão urbana foram atenuados devido as áreas de proteção ambiental.
\end{abstract}

Palavras-chave: Land surface temperature; Degradação ambiental; Normalized difference vegetation index; Nordeste do Brasil.

\footnotetext{
Abstract

The growth of cities, along with the disorderly formation of large metropolises around the world, results in major changes in Land Use. However, few studies relate the urban sprawl and its effects on the cities of the Northeast of Brazil (NEB). Thus, this study aimed to evaluate the urban sprawl in Maceió-Alagoas between 1985 to 2020 from orbital Land Surface Temperature (LST) and Normalized Difference Vegetation Index (NDVI) products to detect the changes and their environmental effects. This study used orbital products acquired from the systems-sensors Landsat 5 / Thematic Mapper (TM) and 8 / Operational Land Imager (OLI). The study used four images to observe the Spatiotemporal variation of urbanization, corresponding to the years 1987, 1998, 2006, and 2020. It used the environment software R for the generation of NDVI and LST thematic maps. The results obtained pointed out substantial changes in the Land Use detected by the NDVI and increased LST over the 35 years. Such variability occurred in the neighborhoods located in the northern and northwestern portion of the city, resulting from the incentive programs of the Federal Government in 2010, mainly the Benedito Bentes Complex (CBB), with the most significant transformations in the use and occupation of the soil, mainly the largest increase in LST between $7.5-10.0^{\circ} \mathrm{C}$. The effects produced by urban sprawls have been mitigated due to the environmental protection areas.
} 
Keywords: Land surface temperature; Environmental degradation; Normalized difference vegetation Index; Northeast of Brazil.

\begin{abstract}
Resumen
El crecimiento de las ciudades junto con la formación desordenada de grandes metrópolis alrededor del mundo da como resultado cambios importantes en el uso y ocupación del suelo. Sin embargo, son pocos los estudios que relacionan la expansión urbana y sus efectos sobre las ciudades del Nordeste de Brasil (NEB). Así, el objetivo de este estudio fue evaluar la expansión urbana en Maceió-Alagoas entre 1985 a a 2020 a partir de productos orbitales de Land Surface Temperature (LST) e Normalized Difference Vegetation Index (NDVI), con el propósito de detectar los cambios y sus efectos ambientales. Para ello se utilizaron productos orbitales adquiridos a partir de los sistemassensores Landsat 5/Thematic Mapper (TM) y 8/Operational Land Imager (OLI). En el estudio se utilizaron cuatro imágenes para la observación de la variación espacio-temporal de la urbanización, correspondiente a los años 1987.1998, 2006 y 2020. Los mapas temáticos de NDVI y LST se generaron a partir del software de medio ambiente R. Los resultados obtenidos señalaron cambios sustanciales en el uso y ocupación del suelo detectado por el NDVI, y aumento de LST en los 35 años. Tal variabilidad ocurrió en los barrios ubicados en la porción norte y noroeste de la ciudad, producto de los programas de incentivos del Gobierno Federal en el 2009, principalmente el Complejo Benedito Bentes (CBB) con las mayores transformaciones en el uso y ocupación del suelo, principalmente el mayor incremento en LST entre 7,5-10, $0^{\circ} \mathrm{C}$. Los efectos producidos por las redes urbanas se han mitigado debido a las áreas de protección ambiental.
\end{abstract}

Palabras clave: Land surface temperature; Degradación ambiental; Normalized Difference vegetation index; Noreste de Brasil.

\title{
1. Introdução
}

Nas últimas décadas, ocorreram profundas transformações socioeconômicas e geoambientais nas cidades brasileiras com população superior a 500 mil habitantes (Maricato et al., 2018; IBGE, 2020). Parte dessas transformações se deve a implantação de políticas públicas Federais com intuito do desenvolvimento e expansão das cidades, destaque para os Programas Aceleração do Crescimento (PAC) e o Minha Casa Minha Vida (PMCMV) - (Menezes \& Mourão, 2017; Correia Filho et al., 2019). No entanto, tais políticas públicas no Brasil não ocorreram de maneira coordenada e planejada e, assim resultaram em mudanças drásticas no uso e ocupação do solo (Freitas, 2014; Correia Filho et al., 2019) e no clima local e regional (Marengo, 2014). Esse processo intensivo de urbanização e de reurbanização causaram alterações significativas no ambiente natural, com a supressão da vegetação nativa por materiais e equipamentos urbanos e no aumento da sensação de desconforto ambiental (Silva, 2001; Deparis, 2014; Gobo et al., 2018; Correia Filho et al., 2019).

Vale destacar que estes efeitos também contribuíram para o aumento das emissões atmosféricas de gases de efeito estufa (GEE) e na piora da qualidade do ar das regiões metropolitanas (RM) do Brasil - (Zeri et al., 2016), e em situações severas, problemas de saúde pública (Guha et al., 2018; Maricato et al., 2018). É sabido com o desenvolvimento das cidades, ocorre impermeabilização de solos, seguido da ocorrência de inundações repentinas e alagamento em áreas urbanas, que por sua vez resultam em problemas de drenagem urbana que causam aumento das doenças de veiculação hídrica (dengue e leptospirose) - (Correia Filho, 2017; Santos et al., 2018; Oliveira Júnior et al., 2019). Estes episódios endêmicos têm sido constatados e recorrentes no mundo (Li et al., 2014; Guha et al., 2018) e no Brasil (Alves, 2017; Correia Filho et al., 2019; Sousa \& Martins Filho, 2019).

Na literatura científica utilizam-se os produtos de Sensoriamento Remoto (SR) na avaliação das transformações oriundas do processo de urbanização, por exemplo, o Normalized Difference Vegetation Index (NDVI) e a Land Surface Temperature (LST) - (Li et al., 2014; Guha et al., 2018; Correia Filho et al., 2019; Pereira et al., 2019). O NDVI é o índice mais utilizado na avaliação da dinâmica da vegetação (Dagnachew et al., 2020), devido a sua capacidade de detectar alterações na cobertura vegetal com rapidez e baixo custo operacional para estudos de alterações climáticas urbanas e degradação ambiental (Lopes et al., 2010; Guha et al., 2018; Correia Filho et al., 2019; Silva et al., 2021). A temperatura da superfície (LST) durante e após a urbanização é investigada via Sensoriamento Remoto (SR) - (Li et al., 2014; Pal \& Ziaul, 2017; Guha et al., 2018; Alves et al., 2019; Correia Filho et al., 2019). A LST é afetada pelas mudanças climáticas, e sua estimativa é útil 
na avaliação da urbanização, desastres naturais e processos de desertificação, sendo um importante indicador de degradação ambiental e mudanças climáticas (Oliveira, 2012; Li et al., 2014; Guha et al., 2018).

Assim como na maioria das capitais brasileiras, nas últimas quatro décadas, Maceió, capital de Alagoas, na região Leste do Nordeste do Brasil (ENEB), também passou por um rápido processo de urbanização associada a problemas em infraestrutura e sistemas de ocupação urbana (Silva et al., 2018; Santiago \& Gomes, 2019; Correia Filho et al., 2019) e, recentemente devido ao acidente geológico provocado pela mineração Braskem ocorreu uma nova reurbanização da cidade. Quando se trata da avaliação dos efeitos da expansão urbana nos bairros de Maceió, há poucos estudos relativos a este assunto (Araújo \& Di Pace, 2010; Santiago \& Gomes, 2016; Correia Filho et al., 2019) e não há um estudo que mostre os impactos socioambientais a partir da expansão urbana. Portanto, o objetivo deste estudo é avaliar a expansão urbana a partir dos produtos de SR na Cidade de Maceió no período de 1985 e 2020, e destes impactos no aspecto socioambiental.

\section{Metodologia}

Maceió, capital do estado de Alagoas (Figura 1), possui uma área de 509,320 km², com uma população estimada em 1,025 milhão de habitantes (IBGE, 2021). Segundo a classificação de Köppen-Geiger é categorizado como tropical quente e úmido 'As', com variações na amplitude térmicas ao longo do ano (Correia Filho et al., 2019). As temperaturas médias variam entre $25,0^{\circ} \mathrm{C}$ a $26,4^{\circ} \mathrm{C}$, com mínimas e máximas de $19,0^{\circ} \mathrm{C}$ e $31,0^{\circ} \mathrm{C}$, respectivamente. Maceió registra valores anuais de chuva entre 1.500 e $2.000 \mathrm{~mm}$, com estações bem definidas: i) seca, entre os meses de setembro a fevereiro; ii) chuvosa, entre os meses de março a agosto (Correia Filho et al., 2019; Oliveira-Júnior et al., 2021).

Atualmente, o Plano Diretor da Cidade de Maceió atual exibe que a área urbana de Maceió é constituída por 50 bairros, subdividida em em oito regiões administrativas (RA), ilustrada na Figura 1 e a Tabela 1 (PMAS-MACEIÓ, 2014).

Tabela 1. Lista dos Bairros por Região Administrativa no município de Maceió.

\begin{tabular}{c|l}
\hline $\begin{array}{c}\text { Região } \\
\text { Administrativa (RA) }\end{array}$ & \multicolumn{1}{c}{ Bairros } \\
\hline RA1 & $\begin{array}{l}\text { Poço, Jaraguá, Ponta da Terra, Pajuçara, Ponta Verde, Jatiúca e Mangabeiras. } \\
\text { Centro, Pontal da Barra, Trapiche da Barra, Prado, Levada, Vergel do Lago, Ponta Grossa. } \\
\text { RA2 }\end{array}$ \\
RA3 & $\begin{array}{l}\text { Farol, Pitanguinha, Pinheiro, Gruta de Lourdes, Canaã, Santo Amaro, Jardim Petropólis, } \\
\text { Bom Parto, Mutange, Bebedouro, Chã de Bebedouro, Petropólis, Chã da Jaqueira, Santa } \\
\text { Amélia, Fernão Velho e Rio Novo. }\end{array}$ \\
RA5 & $\begin{array}{l}\text { Jacintinho, Barro Duro, Serraria, São Jorge e Feitosa. } \\
\text { RA6 }\end{array}$ \\
RA7 & $\begin{array}{l}\text { Santos Dumont, Cidade Universitária, Santa Lúcia, Tabuleiro dos Martins e Clima Bom. } \\
\text { RA8 }\end{array}$ \\
\hline
\end{tabular}

Fonte: PMAS-MACEIÓ (2014). 
Figura 1. Bairros do município de Maceió/AL e suas 8 regiões administrativas (RA).

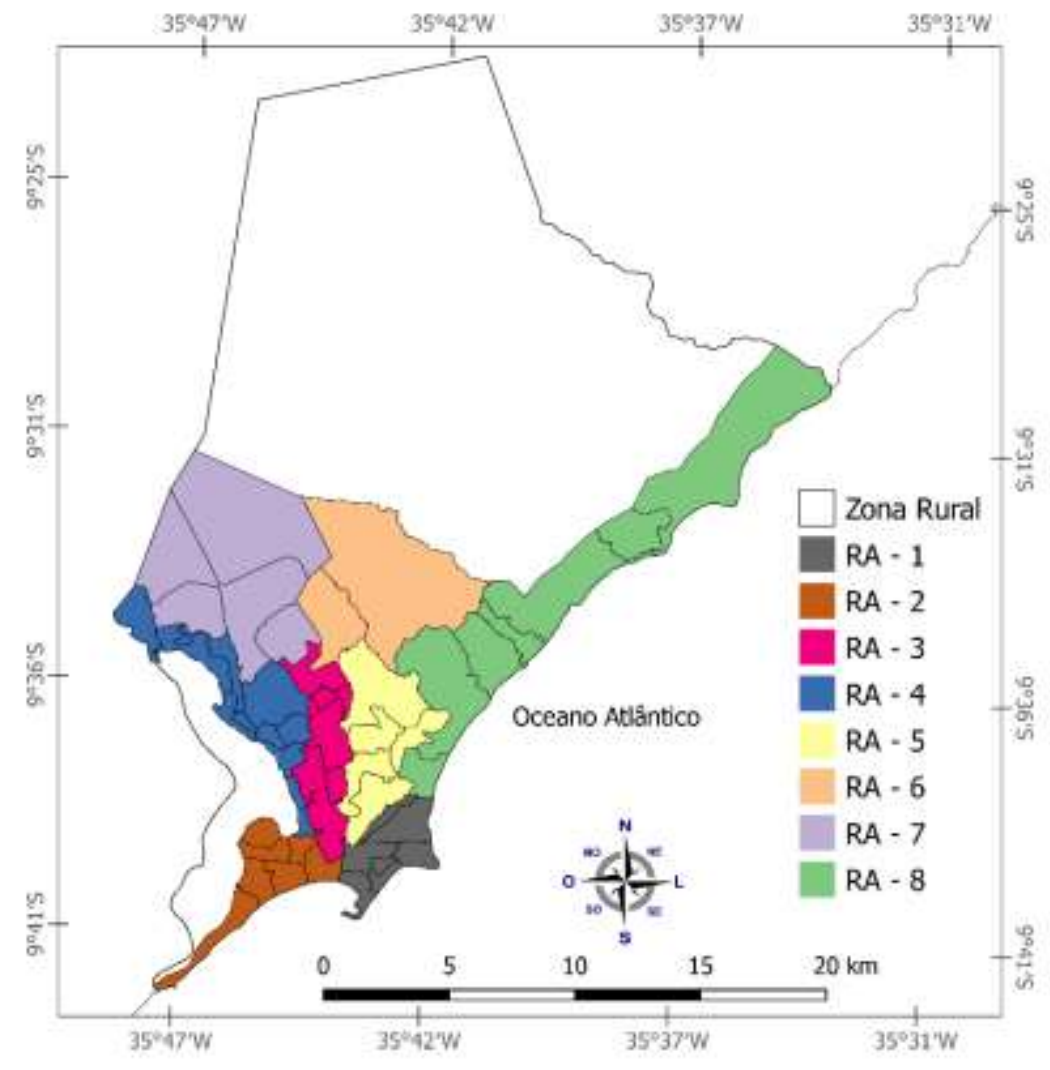

Fonte: PMAS-MACEIÓ (2014). Mapa elaborado pelos autores.

\subsection{Dados}

Para a avaliação da expansão urbana dos bairros Maceió foram utilizadas imagens dos sistemas-sensores Landsat 5/Thematic Mapper (TM) e 8/Operational Land Imager (OLI), adquiridas a partir do endereço eletrônico https://earthexplorer.usgs.gov/, gerenciado pela United States Geological Survey - USGS (USGS, 2021). A partir disso, selecionaram-se quatro imagens com o mínimo de presença de nuvens, sendo avaliada a espaço-temporalidade da urbanização ao longo dos 35 anos (1985-2020), descritas na Tabela 2. As imagens foram submetidas às seguintes etapas de: 1) organização; 2) pré-processamento; 3) manipulação e extração dos valores de LST e NDVI; 4) Avaliação dos mapas temáticos. Todas as etapas foram realizadas no software ambiente R versão 3.4.1 (R Core Development Team, 2019).

Tabela 2. Data de aquisição da imagem orbital, ângulo de elevação (ELV, em $\left.{ }^{\circ}\right)$ e azimute solar $\left(\mathrm{AZS}\right.$, em $\left.{ }^{\circ}\right)$, cobertura de nuvens (CN em \%), e distância Terra-Sol (DTS) das imagens Landsat 5 and 8.

\begin{tabular}{c|c|c|c|c|c}
\hline Path/Row & Data & ELV $\left(^{\circ}\right)$ & AZS $\left(^{\circ}\right)$ & CN (\%) & DTS \\
\hline \multirow{3}{*}{$\mathbf{2 1 4 / 0 6 7}$} & $07 / 09 / 1987$ & 56,28 & 113,15 & 9,00 & 0,98 \\
\cline { 2 - 6 } & $21 / 09 / 1998$ & 56,41 & 73,13 & 23,00 & 1,00 \\
\cline { 2 - 6 } & $26 / 08 / 2006$ & 53,43 & 56,84 & 11,00 & 1,01 \\
\cline { 2 - 6 } & $14 / 10 / 2020$ & 65,88 & 92,50 & 20,81 & 0,99 \\
\hline
\end{tabular}

Fonte: USGS (2021). 


\subsection{Obtenção do NDVI}

Para a extração dos dados de NDVI foram realizadas algumas etapas subsequentes. A primeira é o pré-processamento das bandas espectrais obtidas pelos satélites-sensores Landsat 5/TM e 8/OLI, respectivamente, seguido da conversão do número digital $(N D)$ em valores de radiância e reflectância monocromáticas. As bandas utilizadas para cada índice são descritas na Tabela 3.

Tabela 3. Descrição das bandas do TM, OLI e TIRS do Landsat 5 e 8, com os respectivos coeficientes de calibração $\left(a_{i}\right.$ e $\left.b_{i}\right)$ e irradiação solar espectral $\left(E_{\lambda i}\right)$.

\section{Landsat 5}

\begin{tabular}{c|c|c|c}
\hline Descrição das bandas TM & Comprimento de onda & \multicolumn{2}{|c}{ Coeficientes de calibração } \\
\cline { 3 - 4 } & & $\boldsymbol{a}_{\boldsymbol{i}}$ & $\boldsymbol{b}_{\boldsymbol{i}}$ \\
\hline Band 3 - Red & $0,63-0,69$ & $-1,17$ & 264,00 \\
\hline Band 4 - Near Infrared (NIR) & $0,79-0,90$ & $-1,51$ & 221,00 \\
\hline Band 5 - Shortwave Infrared (SWIR 1) & $1,55-1,75$ & $-0,37$ & 30,20 \\
\hline Banda 6 - Thermal & $10,40-12,50$ & 1,24 & 15,30 \\
\hline
\end{tabular}

\section{Landsat 8}

\begin{tabular}{c|c|c|c}
\hline \multirow{2}{*}{ Descrição das bandas OLI } & \multirow{2}{*}{ Comprimento de onda } & \multicolumn{2}{|c}{ Coeficientes de calibração } \\
\cline { 2 - 4 } & & $\boldsymbol{a}_{\boldsymbol{i}}$ & $\boldsymbol{b}_{\boldsymbol{i}}$ \\
\hline Band 4 - Red & $0,636-0,673$ & $-51,54$ & 624,17 \\
\hline Band 5 - Near Infrared (NIR) & $0,851-0,879$ & $-31,54$ & 381,96 \\
\hline Band 6 - Shortwave Infrared (SWIR 1) & $1,566-1,651$ & $-7,84$ & 94,99 \\
\hline Band 10 - Thermal Infrared (TIRS 1) & $10,60-11,19$ & 0,10 & 22,00 \\
\hline
\end{tabular}

Fonte: USGS (2021).

A conversão do $N D$ em radiância espectral do topo da atmosfera (TOA) foi obtida pela Eq. 1 (Guha et al., 2018):

$\rho_{d i}{ }^{f}=a_{i}+\frac{b_{i}-a_{i}}{255} * N D_{\text {s }}$ for Landsat 5 images

Or

$\rho_{d i}{ }^{f}=a_{i}+\frac{b_{i}-a_{i}}{65585} * N D$, for Landsat 8 images

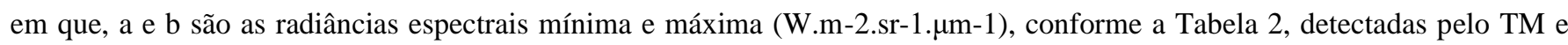
OLI da série Landsat; i corresponde às bandas 3, 4, 5 e 6 (banda termal) do sensor TM do Landsat 5 (bandas 4, 5 e 6 do sensor OLI, e as bandas 10 e 11 do sensor Thermal Infrared Sensor (TIRS) do Landsat 8).

Em seguida foi calculado a reflectância monocromática de cada banda ( $\left.\rho_{\lambda_{i}}\right)$, definida como a razão entre o fluxo de radiação refletido e o fluxo de radiação solar incidente, foi obtida segundo a Eq. 2 (Allen et al., 2002):

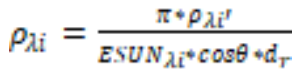


em que, $E S U N_{\lambda i}$ é a irradiância solar espectral de cada banda no topo da atmosfera $\left(\mathrm{W} \cdot \mathrm{m}^{-2} \cdot \mathrm{sr}^{-1} \cdot \mu \mathrm{m}^{-1}\right)$, apresentada na Tabela 2 , $\theta$ é o ângulo zenital solar e $d_{r}$ é a distância Terra-Sol.

Após a obtenção da radiância e reflectância monocromática, buscou-se a obtenção do NDVI, a partir da reflectância das bandas NIR ( $\left.\rho_{\text {NIR }}\right)$ e vermelha $\left(\rho_{R}\right)$. O principal objetivo do NDVI é identificar a atividade fotossintética da vegetação numa determinada região, mas é comumente utilizada em diferentes abordagens, incluindo a detecção de Ilha de Calor Urbano (ICU) e a expansão urbana (Santiago \& Gomes, 2016; Correia Filho et al., 2019).

$N D V I=\frac{\left(\rho_{N I K}-\rho_{W}\right)}{\left(\rho_{N I K}+\rho_{E}\right)}$

em que, $\rho$ NIR e $\rho R$ correspondem, respectivamente, às reflectâncias das bandas do infravermelho-próximo e vermelho. Os valores de NDVI variam de -1 a 1 , onde os valores positivos correspondem a áreas com vigor vegetativo (superfície terrestre) de acordo com a atividade fotossintética da vegetação e sua densidade na área considerada (pixel); enquanto os valores negativos refletem massas de água e a presença de nuvens (Rouse, 1974; Guha et al., 2018; Correia Filho et al., 2019). Desta maneira, o NDVI foi classificado em cinco classes: Corpos d'água / Nuvens, solo nu / área construída, vegetação esparsa, vegetação abundante e vegetação densa, descrito na Tabela 4.

Tabela 4. Classificação do NDVI em cinco classes de uso e ocupação do solo, com os seus respectivos intervalos.

\begin{tabular}{c|c}
\hline Classes & Intervalos \\
\hline Corpos d'água / nuvens & $-1,00$ a $-0,20$ \\
Solo nu / Área construída & $-0,20$ a 0,15 \\
Vegetação esparsa & 0,15 a 0,40 \\
Vegetação abundante & 0,40 a 0,60 \\
Vegetação densa & 0,60 a 1,00 \\
\hline
\end{tabular}

Fonte: Correia Filho et al. (2021).

\subsection{Temperatura da Superfície Terrestre (LST)}

A obtenção do LST é a partir das seguintes etapas, o cálculo da emissividade da superfície terrestre extraídos do NDVI, a partir da metodologia proposta de Guha et al. (2018) pela Eq.4:

$d_{g}=\left(1-\varepsilon_{g}\right) *\left(1-P_{V}\right) * F * \varepsilon_{V}$

$\varepsilon_{\mathrm{v}}$ é a emissividade da vegetação, $\varepsilon_{\mathrm{s}}$ é a emissidade do solo, $\mathrm{F}$ é o fator médio (valor igual a 0,55 ), $\mathrm{P}_{\mathrm{v}}$ é a proporção da vegetação obtida pela seguinte Eq. 5, descrita abaixo:

$P_{D}=\left(\frac{\text { NDVI }-N D V I_{\text {min }}}{N D V I_{\max }+N D V I_{\text {minin }}}\right)^{2}$

Para a obtenção de emissidade é obtida pela Eq.6:

$\varepsilon=\varepsilon_{D} * P_{D}+\varepsilon_{g} *\left(1-P_{D}\right)+d_{g}$

Para obter o LST é utilizada a banda térmica com dados de radiância monocromática, que são calculados e convertidos em temperatura de brilho. O LST é um dos principais indicadores do efeito da urbanização, resultante da alteração do microclima local em virtude da mudança no Uso e Ocupação do Solo (De Souza et al., 2016; Guha et al., 2018; Correia Filho et al., 2019). Para isto, é necessário calcular a radiância monocromática (Eq. 2) da banda 6 do sensor TM do Landsat 
5/TM (Bandas 10 do sensor TIRS1 para o Landsat 8/OLI). Após a obtenção da radiação espectral, calculou-se a BT pela Eq. 7 , e posteriormente a LST pela Eq. (8) - (Guha et al., 2018):

$$
T B=\left(\frac{K_{2}}{\ln \left(\frac{K_{2}}{P_{\lambda \mathrm{i}}}+1\right)}\right)-273,16 \quad(7) \quad L S T=\frac{T B}{1+W *\left(\frac{T B}{P}\right) * \ln (\varepsilon)}
$$

Em que, $T B$ é a temperatura de brilho dada em Celsius $\left({ }^{\circ} \mathrm{C}\right)$, para o satélite Landsat 5 os valores de $K_{1}$ e $K_{2}$ correspondem a $607.76 \mathrm{~W} \cdot \mathrm{m}^{-2} \cdot \mathrm{sr}^{-1} \cdot \mu \mathrm{m}^{-1}$ e $1260.56 \mathrm{~K}$, e o Landsat 8 foram de $774.88 \mathrm{~W} \cdot \mathrm{m}^{-2} \cdot \mathrm{sr}^{-1} \cdot \mu \mathrm{m}^{-1}$ e de $1321.08 \mathrm{~K}$, respectivamente. $W$ é o comprimento de onda efetiva $(11.475 \mu \mathrm{m}), \mathrm{P}=\mathrm{h} * \mathrm{c} / \mathrm{s}\left(1.438 * 10^{-2} \mathrm{mK}\right), h=$ Constante de Planck, $c=$ velocidade da luz, $s=$ constante de Boltzmann $\left(1.38 * 10^{-23} \mathrm{~J} / \mathrm{K}\right)$ e $\varepsilon$ é a emissividade da superfície terrestre.

\section{Resultados e Discussão}

\subsection{NDVI}

A avaliação da cobertura vegetal da cidade de Maceió via NDVI (Figura 2) mostrou uma intensa mudança no uso e ocupação do solo na cidade, principalmente nas porções norte e nordeste (RA6) e noroeste (RA7) - (Tabela 1). Com base nas imagens selecionadas (Tabela 2), uma comparação entre o final (2020) e o início da série temporal (1987), aponta mudanças marcantes na parte da alta da cidade e na região próxima ao litoral norte, motivada pela especulação imobiliária, migração forçada devido ao acidente da Braskem, enchentes e deslizamentos e degradação dos bairros históricos de Maceió (Nascimento et al., 2018).

Figura 2. Avaliação espacial do uso e ocupação do solo na cidade de Maceió via NDVI, durante os anos de 1987, 1998, 2006 e 2020.

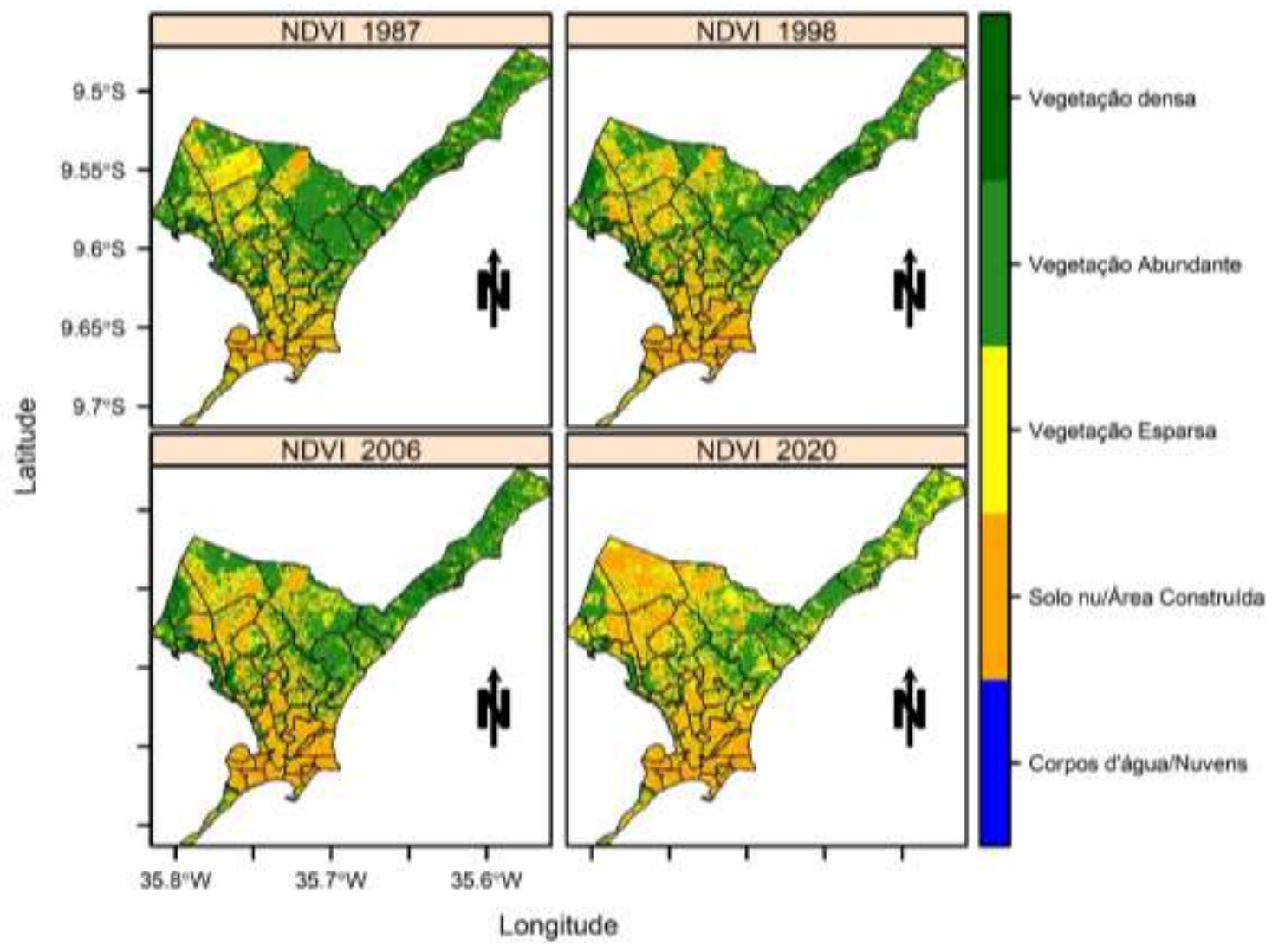

Fonte: USGS (2021). Mapa elaborado pelos autores. 
Os percentuais das categorias obtidas do NDVI mostraram uma diminuição significativa nas áreas com vegetação $(62,7 \%)$ - (Tabela 4), isso é preocupante, pois exibe a supressão da vegetação da Mata Atlântica, bioma extremamente ameaçado e que tem relação diretamente com dinâmica da chuva no NEB (Correia Filho et al., 2019). Vale destacar que a maior concentração de NDVI com valores negativos, novamente ocorreram nas RA6 e RA7, com 10,7\%, sendo associada diretamente com a expansão urbana e identificadas em estudos anteriores (Araújo \& Di Pace, 2010, Santiago \& Gomes, 2016) e recente (Correia Filho et al., 2019).

O processo de migração populacional em Maceió nas regiões sul para o norte, noroeste e nordeste se deve em parte à saturação de edifícios residenciais e comerciais, então a única maneira foi expandir para o norte e nordeste da cidade (Santiago \& Gomes, 2016). Por outro lado, a expansão para o norte e nordeste de Maceió foi uma alternativa para minimizar a vulnerabilidade de Maceió, no entanto, parte desta expansão em Áreas de Preservação Ambiental (APA) do Catolé e Fernão Velho, localizada entre as cidades de Maceió (região oeste da área de estudo) e Satuba. Esta APA é uma unidade de conservação sob orientação do Instituto do Meio Ambiente (IMA) e a Companhia de Saneamento de Alagoas (CASAL), e protegida pelo Batalhão de Polícia Ambiental (BPA), ao qual possui papel estratégico para a cidade de Maceió, responsável por 30\% do sul e sudeste da cidade de Maceió, mantidos pelos riachos Aviação e Catolé (Moura, 2014; IMA, 2019).

Comparativamente, entre os anos de 1987 e 2020 (Figura 2) a área construída com o maior percentual (RA7) (de 17,8\% para 51,8\%) aumentou 34\% no período de estudo, devido à expansão dos conjuntos habitacionais, da categoria de solo exposto e corredores viários, sendo intensificados a partir da execução dos Programas do Governo Federal (PAC e PMCMV) (Carvalho, 2015; Correia Filho et al., 2019). Vale ressaltar que a redução gradativa da categoria de vegetação (redução de área de 30,4\% para 10,7\%) ocorreu pela expansão para o norte e noroeste da cidade de Maceió que modificou gradativamente toda a paisagem devido ao grau de vulnerabilidade físico-ambiental das zonas sul e sudeste de Maceió motivado pelas chuvas intensas, que resultam em enchentes e deslizamentos na região em décadas (Nascimento et al., 2018; Oliveira-Júnior et al., 2021).

O ano de 1987 exibe predominância das classes vegetação esparsa, abundante e densa nas porções centro-norte e noroeste da cidade de Maceió, correspondente as RA6 e RA7 (Figura 1), ao contrário, da porção centro-sul com o predomínio da classe área construída/solo nu. Em 1998, houve diminuição significativa na vegetação nas porções Norte e Noroeste da cidade de Maceió, sendo associada à expansão da cidade. Já na porção Oeste, localizada na região da APA localizada entre os bairros Clima Bom (RA7), Fernão Velho (RA4) e Santos Dumont (RA7) - (Tabela 1), houve uma diminuição, segundo o NDVI.

A partir do ano 2009, iniciou-se a construção de diversos conjuntos habitacionais no Complexo do Benedito Bentes (CBB), porção norte da cidade, por exemplo, os conjuntos Cidade Sorriso I e II entre outros, a fim de solucionar alguns problemas do déficit habitacional, assim como a remoção da população de áreas de vulnerabilidade social, tais como: Favela de Jaraguá, Cidade de Lona (Eustáquio Gomes), nas favelas Sururu de Capote e Torre (Orla Lagunar), do Canaã e River Plate (no Tabuleiro do Martins). A partir dos novos conjuntos habitacionais, houve maior concentração de área construída na parte alta da cidade, principalmente nos bairros Cidade Universitária e Benedito Bentes (Correia Filho et al., 2019). Os dois bairros citados anteriormente apresentam alta densidade populacional, desenvolvimento urbano desordenado e baixo investimento em serviços de infraestrutura urbana.

Em 2020, verifica-se que essas alterações ocorreram em toda Maceió, destaque para os bairros Cidade Universitária, Benedito Bentes, Tabuleiro dos Martins e Clima Bom, situados nas RA6 e RA7 (Tabela 1). Destaque para o Benedito Bentes, com o predomínio de vegetação densa e abundante da Mata Atlântica, após implantação dos planos federais associados à necessidade de expansão urbana resultou em mudanças profundas na região, ao qual o Bairro Clima Bom mostrou alta 
variabilidade no NDVI $(0,15$ em 1987) para (-0,20 em 2020). Os resultados obtidos corroboram com estudo realizado por Correia Filho et al. (2019).

Segundo Almeida \& Neto (2015), em 1988 indústrias no Distrito Industrial (situado nas porções Noroeste e Norte, no RA6), que contribuiu na expansão urbana nos bairros adjacentes. No ano de 2002, antigas áreas ocupadas, no bairro do Tabuleiro do Martins, intensificaram as áreas impermeabilizadas com a expansão de novos loteamentos e edificações adjacentes aos bairros Cidade Universitária, Clima Bom e Santos Dumont.

Em meados da década de 2000, houve diversas mudanças no uso e ocupação do solo na porção Norte-Noroeste correspondentes as RA6 e RA7 da cidade de Maceió, acentuados após a implementação dos programas do Governo Federal (PMCMV e PAC) em 2008 (Correia Filho et al., 2019). Por conta disso, houve a instalação de novos comércios locais, tais como, lojas, shoppings e locais de lazer. Como resultado dessa transformação, houve a substituição de vegetação esparsa, abundante e densa para solo nu / área construída. O aumento da população urbana tem levado à expansão do território da cidade, com a construção de casas e prédios e a abertura de novas ruas, sem medidas compensatórias, inicia-se o processo de alteração da paisagem e formação de áreas de degradação, com perda e fragmentação da cobertura vegetal, alteração de habitats naturais, micro-habitat, processos biológicos, perturbação da flora e da fauna e afastamento de animais são caraterísticas marcantes dessas alterações decorrentes das alterações do uso e ocupação do solo (Deparis, 2014).

O PAC contribuiu para o crescimento populacional e a industrialização associada às mudanças nos costumes (valores) que exacerbaram significativamente as mudanças climáticas, especialmente em áreas urbanas e outras áreas locais. Diferentes atividades humanas nos espaços internos das áreas de urbanização (parques, indústrias, edifícios etc.), combinadas com a configuração e distribuição desses espaços, podem promover sobremaneira as mudanças climáticas e configurar diferentes categorias de microclimas (Buccheri-Filho \& Tonetti, 2011). O PMCMV foi um dos programas habitacionais implantados pelo governo brasileiro que permitiu aos cidadãos sonharem em ter casa própria, criando muitas oportunidades de emprego e promovendo o desenvolvimento econômico local. O objetivo é ampliar o acesso à moradia digna por ações integradas de padronização de cidades, terrenos e segurança, habitação, saneamento e inclusão social, e permitir que os moradores vivessem ou se reassentem permanentemente quando houver risco permanente (Brasil, 2009).

Belló (2004) afirmou anteriormente que a urbanização sem o planejamento adequado pode ocasionar efeitos catastróficos sob o ponto de vista ambiental e, assim aumenta a degradação ambiental. De acordo com Lima (2011), as cidades constituem uma forma intensa de transformação da paisagem natural, cujos impactos não se limitam a mudar a morfologia do terreno no qual se inserem, mas também, as condições ambientais e climáticas. Anteriormente Lombardo (1985) mostrou que a urbanização ao nível do espaço físico da edificação alterou significativamente o clima urbano, tendo em conta o aumento da área de absorção de calor, impermeabilização dos solos, alterações da cobertura vegetal e falhas, com a construção de edifícios que perturbam os efeitos do vento.

Em geral, a mudança no uso e ocupação do solo de vegetação relativa à área construída apresentou um aumento significativo em 1998, iniciando a ocupação na direção norte-nordeste da cidade de Maceió. Em 2006 e 2017, o NDVI apontou que toda a parte alta de Maceió foi ocupada por construções ou solo nu. As regiões classificadas como vegetação densa/abundante foram substituídas por ocupações urbanas, com a ampliação de construções de novos conjuntos habitacionais e empreendimentos comerciais, que por sua vez alterou os microclimas das regiões (Santiago \& Barros, 2016; Nascimento et al., 2018; Correia Filho et al., 2019). Vale destacar que a urbanização é crescente e global e, nas últimas décadas, as cidades apresentaram crescimento significativo da população, do espaço e de atividades e, assim transformou rapidamente ambos os ambientes naturais e construído. Este ambiente recém-construído tem sido impactado pelas mudanças climáticas, que prejudicam a qualidade de vida das pessoas (Labaki et al., 2011; Oliveira et al., 2013). 


\subsection{LST}

A Figura 3 exibe a variabilidade espacial da LST na cidade de Maceió. Durante os anos de 1987, 1998 e 2006, a LST variou entre $22,5^{\circ} \mathrm{C}$ a $32,5^{\circ} \mathrm{C}$, sendo que as diferenças não ultrapassaram $7,5^{\circ} \mathrm{C}$, exceto quando a LST foi inferior a $22,5^{\circ} \mathrm{C}$, devido à presença de nuvens. Tais diferenças identificadas no estudo se devem aos empreendimentos comerciais e habitacionais na área de estudo que nas últimas décadas aumentou consideravelmente (Santiago \& Barros, 2016; Correia Filho et al., 2019). Destaque para os bairros situados no sul da cidade, neste caso os bairros do Centro e Farol, situados na RA2 e RA3, respectivamente, com os maiores registros de LST entre $27,5^{\circ} \mathrm{C}-32,5^{\circ} \mathrm{C}$.

Figura 3. Avaliação temporal da LST $\left({ }^{\circ} \mathrm{C}\right)$ na cidade de Maceió via NDVI, durante os anos de 1987, 1998, 2006 e 2020.

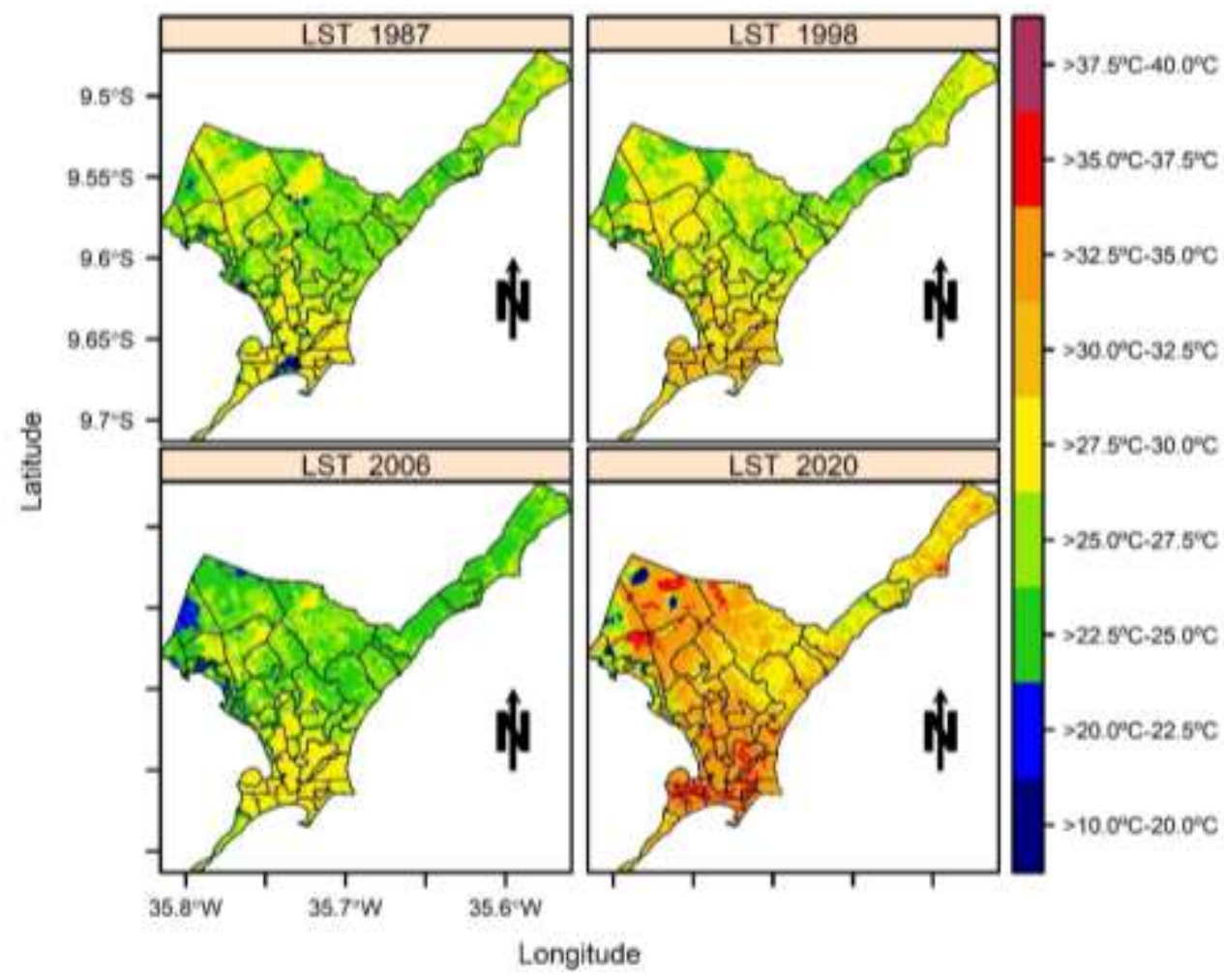

Fonte: USGS (2021). Mapa elaborado pelos autores.

Os anos de 1987 e 1998 apresentaram um aumento considerável no LST, na faixa entre $22,5^{\circ} \mathrm{C}$ e $25,0^{\circ} \mathrm{C}$ para $27,5^{\circ} \mathrm{C}$ e $30,0^{\circ} \mathrm{C}$, com um aumento percentual de $45,0 \%$ para $60,0 \%$ (RA7). Tal aumento está relacionado à substituição da classe de vegetação pela classe de área construída (asfalto ou concreto) ou solo descoberto, que retém maior quantidade de calor (Santiago \& Gomes, 2016). Anteriormente, Araújo \& Di Pace (2010) avaliaram o LST na cidade de Maceió entre 1990 e 2003 , sendo identificado que os maiores valores de LST ocorreram entre 1998 e 2003, respectivamente.

No ano de 2020, novamente houve aumento significativo na LST em relação aos anos anteriores, entre $32,5^{\circ} \mathrm{C}$ e $37,5^{\circ} \mathrm{C}$ na porção noroeste $(\mathrm{NW})$ da cidade, com percentuais superiores a $50,0 \%$, sendo uma variação entre $7,5-10,0^{\circ} \mathrm{C}$. Tal aumento na LST está relacionado à substituição da classe de vegetação por categorias de área construída (asfalto ou concreto) ou solo descoberto em Maceió, que retém maior quantidade de calor (Santiago \& Gomes, 2016, Alves et al., 2019).

Outro fator que resultou no aumento na LST foi à migração populacional de outras cidades para capital Maceió, principalmente após os planos PAC e PMCMV, que sucedeu na implementação de uma série de empreendimentos nas regiões mencionadas com a instalação de shopping, conjuntos habitacionais, faculdades e fábricas (Santiago \& Gomes, 2016; Correia Filho et al., 2019). Em proporção inferior às enchentes e inundações, porém em número de ocorrências mais frequentes, os 
eventos relacionados aos deslizamentos de terras também têm somado prejuízos entre os municípios da Região Metropolitana de Maceió (RMM). Embora ao contrário das inundações ou enchentes que fazem parte dos registros locais, não há informações disponíveis sobre os registros históricos de deslizamentos entre áreas metropolitanas, mas é sabe-se que as áreas mais vulneráveis aos movimentos de massa (encostas) estão degradadas ou irregularmente ocupadas por unidades habitacionais instáveis. Portanto, na realidade social e espacial da população da RMM, a vulnerabilidade às chuvas sempre foi constante (Nascimento et al., 2018; Oliveira Júnior et al., 2021).

Em contrapartida, não se detectou tal aumento na LST em outros bairros da cidade, por exemplo, na Chã da Jaqueira e Chã de Bebedouro (ambas situadas na RA4), localizados próximo ao Parque Municipal de Maceió, uma área de APA e próxima a Lagoa Mundaú. Os bairros Santos Dumont e Rio Novo ambas nas regiões RA4 e RA7 também não houve aumento da LST, ambos os bairros fazem parte em áreas de preservação da vegetação do bioma Mata Atlântica, resultado similar ao obtido por Correia Filho et al. (2019) na avaliação da LST na parte alta de Maceió, onde as áreas próximas as APA situada nos bairros mencionados, o aumento variou entre $0,5^{\circ} \mathrm{C}$ e $2,0^{\circ} \mathrm{C}$.

Ribeiro et al. (2012) mostraram que no Distrito Federal a expansão desordenada de sua malha urbana histórica e contínua estrutura urbana, afetou diretamente a supressão da vegetação nativa, seguida do uso e ocupação do solo, e, portanto, afetam diretamente o potencial térmico de diversos locais. O aumento do LST urbano causa desconforto térmico, seguido do aumento da temperatura média da cidade e na intensificação da ilha de calor urbana (ICU) - (Pal \& Ziaul, 2017). É sabido que vegetação densa aumenta a evapotranspiração (ET) e resfria a superfície (Price, 1990; Boegh et al., 1998). De acordo com Lapola et al. (2020), áreas de vegetação protegidas são importantes para o processo de mitigação dos efeitos das mudanças climáticas nas cidades, além de manter o funcionamento do ecossistema, preserva os polinizadores, recursos hídricos e serviços ligados às nossas necessidades básicas e à segurança alimentar. Anteriormente Braz et al. (2018) observaram que em áreas verdes apresentam baixas temperaturas, dando uma ideia incorreta que é influenciada apenas pela vegetação, quando na verdade a baixa temperatura do entorno é influenciada pela baixa densidade de construções.

\section{Considerações Finais}

As mudanças significativas no uso e ocupação do solo em Maceió, com redução das classes vegetação densa e abundante, principalmente no bioma Mata Atlântica são identificadas nos bairros do Benedito Bentes, Cidade Universitária e Clima Bom, correspondentes as R6 e R7 via NDVI. Espacialmente a expansão urbana é destacada nas porções norte e noroeste da cidade de Maceió, devido a instalação de diversos empreendimentos comerciais e residenciais. As alterações no uso e ocupação do solo contribuem para o aumento considerável da LST na cidade de Maceió, novamente destaque para as porções norte e noroeste, com uma formação de gradiente térmico da LST entre $10-15^{\circ} \mathrm{C}$. Partes destas alterações são resultantes dos planos do PAC e PMCMV que visavam à expansão e redução do déficit habitacional e não descarta a migração populacional por oportunidade de emprego e ocasionado por enchentes e cheias que são comuns no Estado de Alagoas.

Como mitigação aos efeitos da LST e da degradação ambiental na cidade de Maceió é sugerido à intervenção paisagística via arborização urbana, comum em diversas cidades brasileiras e do mundo. O processo de arborização urbana contribuiria para o aumento da sensação de conforto e bem-estar da população e, ainda pode preservar a regiões de nascentes que ajudam manutenção dos rios que integram o abastecimento de água da região metropolitana de Maceió.

\section{Agradecimentos}

O primeiro autor agradece ao Fundo de Amparo à Pesquisa do Estado de Alagoas (FAPEAL) pela concessão de Bolsa de Iniciação Científica (PIBIC). O Segundo autor agradece ao Conselho Nacional para Pesquisa e Desenvolvimento Tecnológico (CNPq) pela bolsa Pós-Doutorado Júnior de no 161023/2019-3. O quinto autor é colaboradora do Programa 
Institucional de Bolsa de Iniciação Científica (PIBIC)Fundo de Amparo à Pesquisa do Estado de Alagoas (FAPEAL).

\section{Referências}

Allen, R. G., Tasumi, M., Trezza, R., Waters, R., \& Bastiaanssen, W. (2002). SEBAL (surface energy balance algorithms for land). Advance training and users manual-Idaho implementation, version, 1, 97.

Almeida, A., \& Neto, J. (2015). Análise do processo de urbanização e impermeabilização do solo da bacia endorreica do Tabuleiro do Martins, Maceió-AL. Maceió: Universidade Federal de Alagoas, 12 p.

Alves, E. D. L. (2017). Ilha de Calor Urbana e Simulações: aplicação metodológica para o reordenamento do território. Caminhos de Geografia, 18(61), 3343.

Alves, L. E. R., Correia Filho, W. L. F., Gomes, H. B., Oliveira-Júnior, J. F., \& Sanches, F. O. (2019). Space-Temporal evaluation of Changes in Soil Use and Soil Cover and Temperature in the Metropolitan Region of Baixada Santista. Biosc. J. (Online), 35:1438-1449, 2019.

Araújo, T. L., \& Di Pace, F. T. (2010). Valores instantâneos da temperatura da superfície terrestre na cidade de Maceió-AL utilizando imagens do satélite TM/Landsat 5. Revista Brasileira de Geografia Física, 3(2), 104-111.

Ayoade, J. (1983). Introdução à climatologia para os trópicos. São Paulo: DIFEL. 332p.

Belló, S. L. (2004). Avaliação do impacto da ocupação urbana sobre as características hidrossedimentométricas de uma pequena bacia hidrográfica de encosta. 161f. Dissertação (Mestrado em Engenharia Civil) - Universidade Federal de Santa Maria, Santa Maria, 2004.

Boegh, E., Soegaard, H., Hanan, H., Kabat, P., \& Lesch, L. (1998). A remote sensing study of the NDVI-Ts relationship and the transpiration from sparse vegetation in the Sahel based on high resolution data. Remote Sens. Environ., 69, 224-240.

Brasil. Ministério das Cidades. Instrução Normativa no 33, de 9 de julho de 2009. Programa: Urbanização Regularização e Integração de Assentamentos Precários.

Braz, A. M., Kunkel, A. C., Boni, P. V., Braz, A. M., \& Martins, A. P. (2018). Áreas Verdes e Temperatura da Superfície na Cidade de Três Lagoas/MS. Revista Formação (ONLINE), 25 (45), 93-122.

Buccheri-Filho, A. T., \& Tonetti, E. L. (2011). Qualidade ambiental nas paisagens urbanizadas. Revista Geografar. 6 (1), $23-54$.

Carvalho, H. A. O. A. (2015). Caixa Econômica Federal como agente da política habitacional: um estudo a partir do PAC e PMCMV em Carapicuíba. Dissertação (Mestrado em Teoria e História da Arquitetura e do Urbanismo) - Instituto de Arquitetura e Urbanismo, Universidade de São Paulo, São Carlos. doi: 10.11606/D.102.2015.tde-26082015-105945.

Correia Filho, W. L. F., Santiago, D. B., Oliveira Júnior, J. F., \& Silva Junior, C. A. (2019). Impact of Urban Decadal Advance on Land Use and Land Cover and Surface Temperature in the City of Maceió, Brazil. Land Use Policy, 87, 1-11.

Correia Filho, W. L. F., Santiago, D. B., Oliveira Júnior, J. F., Silva Junior, C. A., Oliveira, S. R. S., Silva, E. B., \& Teodoro, P. E. (2021). Analysis of environmental degradation in Maceió-Alagoas, Brazil via orbital sensors: a proposal for landscape intervention based on urban afforestation. Remote Sensing Applications: Society and Environment. (in press)

Correia, M. F. (2001). Impacto das ações antrópicas no clima do submédio do rio São Francisco: Um estudo numérico e observacional. São Paulo. 181p. /Tese de Doutoramento do Departamento de Ciências Atmosféricas do Instituto de Astronomia, Geofísica e Ciências Atmosféricas da Universidade de São Paulo.

Dagnachew, M., Kebede, A., Moges, A., \& Abebe, A. Effects of Climate Variability on Normalized Difference Vegetation Index (NDVI) in the Gojeb River Catchment, OmoGibe Basin, Ethiopia. Advances in Meteorology, 2020.

Deparis, S. (2014). Implicações no meio ambiente decorrentes da ocupação desordenada na área urbana do município de Concórdia-SC. Diretoria de Pesquisa e Pós Graduação - Especialização em Gestão Ambiental em Municípios. Universidade Tecnológica Federal do Paraná, Medianeira - PR, 40p.

De Souza, D. O., dos Santos Alvalá, R. C., \& do Nascimento, M. G. (2016). Urbanization effects on the microclimate of Manaus: A modeling study. Atmospheric Research, 167, 237-248.

Freitas, C. F. S. (2014). Ilegalidade e degradação em Fortaleza: os riscos do conflito entre a agenda urbana e ambiental brasileira. Revista Brasileira de Gestão Urbana, 6 (1), 109-125.

Gobo, J. P. A., Galvani, E., \& Wollmann, C. A. (2018). Subjective Human Perception of Open Urban Spaces in the Brazilian Subtropical Climate: A First Approach. Climate, 6 (2), 24. https://doi.org/10.3390/cli6020024

Guha, S., Govil, H., Dey, A., \& Gill, N. (2018). Analytical study of land surface temperature with NDVI and NDBI using Landsat 8 OLI and TIRS data in Florence and Naples city, Italy. Eur J. Remote Sensing, 2018 (51), 667-678.

IBGE - Instituto Brasileiro de Geografia e Estatística. (2021). Censo Brasileiro de 2020. Maceió: IBGE.

IMA - Instituto do Meio Ambiente. (2019). Plano de Manejo da Área de Proteção Ambiental do Catolé e Fernão Velho. (Acessado em 08 de Julho 2021). http://www.ima.al.gov.br/wp-content/uploads/2021/01/Plano-de-Manejo-da-APA-do-CatolCA-e-FernCAo-Velho-Estudos-TCAcnicos-e-Zoneamento-

Ambiental.pdf

Jacob, M., Frankl, A., Beeckman, H., Mesfin, G., Hendrickx, M., Guyassa, E., \& Nyssen, J. (2015). North Ethiopian afro-alpine tree line dynamics and forest-cover change since the early 20th century. Land Degradation \& Development, 26(7), 654-664. 
Labaki, L. C., Santos, R. F., Bueno-Bartholomei, C. L., \& Abreu, L. V. (2011). Vegetação e conforto térmico em espaços urbanos abertos. Fórum Patrimônio, $4(1), 23-42$.

Lapola, D. M., Silva, J. M. C. D., Braga, D. R., Carpigiani, L., Ogawa, F., Torres, R. R., \& Joly, C. A. (2020). A climate-change vulnerability and adaptation assessment for Brazil's protected areas. Conservation biology, 34(2), 427-437.

Lima, G. N. Características do Clima Urbano de Nova Andradina - MS. 2011. 161 f. Dissertação (Mestrado em Geografia) - Faculdade de Ciências e Tecnologia, Universidade Estadual Paulista, Presidente Prudente.

Li, L., Tan, Y., Ying, S., Yu, Z., Li, Z., \& Lan, H. (2014). Impact of land cover and population density on land surface temperature: case study in Wuhan, China. Journal of Applied Remote Sensing, 8, 884-993.

Lombardo, M. A. (1985). Ilha de calor nas metrópoles. O exemplo de São Paulo. Hucitec.

Lopes, H., Candeias, A. L. B., Accioly, L. J. O., Sobral, M. C. M. \& Pacheco, A. P. (2010). Parâmetros biofísicos na detecção de mudanças na cobertura e uso do solo em bacias hidrográficas. Revista Brasileira de Engenharia Agrícola e Ambiental, 14 (11), 1210-1219.

Marengo, J. A. (2014). O futuro clima do Brasil. Revista USP, 103, 25-32.

Maricato, E., Colosso, P., \& Comarú, F. D. A. (2018). Um projeto para as cidades brasileiras e o lugar da saúde pública. Saúde em Debate, 42, $199-211$.

Menezes, G., \& Mourão, L. Programa Minha Casa Minha Vida sob a perspectiva da qualidade de vida. Psicoperspectivas, 16, 149-163, 2017.

Moura, J. R. O. (2014). Fotografia da periferia com olhar geográfico: Áreas de riscos na cidade de Maceió/Alagoas/Brasil. Reflexões e Práticas Geográficas, $1,93-113$.

Nascimento, M. C., Lombardo, M. A., Guimarães Júnior, S. A. M., \& Andrade, E. L. (2018). Análise da Vulnerabilidade Físico-Ambiental causada pelas chuvas intensas na Região Metropolitana de Maceió. Caminhos de Geografia, 19, 268-288.

Oliveira, A., Sanches, L., Musis, C., \& Nogueira, M. (2013). Benefits Of Squares In Urban Afforestation - The Case of Cuiabá/MT. Revista Eletrônica em Gestão, Educação e Tecnologia Ambiental, 9(9), 1900-1915. doi:https://doi.org/10.5902/223611707695

Oliveira, L. M. M. (2012). Índice de vegetação e temperatura da superfície no ecônomo ilha do bananal por sensoriamento remoto. Revista de Geografia (Recife), 30 (3), 209-225.

Oliveira-Júnior, J. F., Gois, G., Silva, E. B., Teodoro, P. E., Johann, J., \& Silva Junior, C. A. (2019). Non-parametric tests, multivariate analysis and descriptive and exploratory statistics applied to reported dengue cases in Brazil. Environmental Monitoring and Assessment, 191,473-491.

Pal, S. \& Ziaul, S. (2017). Detection of land use and land cover change and land surface temperature in English Bazar urban centre. Egyptian Journal of Remote Sensing and Space Science, 20 (1), 125-145.

Pereira, R. M., Leão, T. P., Sandri, D., Baptista, G. M. M, \& Cunha, L. S. (2019). Modelagem da Temperatura do Ar na Região do Distrito Federal - Brasil, por meio de Dados Atmosféricos do Sensor AIRS. Revista Brasileira de Meteorologia, 34(2), 275-282.

PMAS - MACEIÓ. (2014). Plano Municipal de Assistência Social de Maceió. http://www.maceio.al.gov.br/wpcontent/uploads/admin/documento/2014/08/PMAS_Macei\%C3\%B3_2014_2017-FINALIZADO-para-upar.pdf

Price, J. C. (1990). Using spatial context in satellite data to infer regional scale evapotranspiration, IEEE Trans. Geosci. Remote Sens., 28,940 - 948.

Ribeiro, R., Holanda, F. \& Coelho, J. (2012). Índices de qualidade configuracional urbana caso do Distrito Federal, Brasil. EURE (Santiago), 38 (114), 229255 .

Rouse, J., Haas, R., Schell, J., Deering, D., \& Harlan, J. (1974). Monitoring the Vernal Advancement of Retrogradation of Natural Vegetation: Final Report. Greenbelt: NASA/GSFC, 371 p.

R Development Core Team. (2019). R: A language and environment for statistical computing version 3.6-4. R Foundation for Statistical Computing, Vienna, Austria, http://www.r-project.org, ISBN 3-900051-07-0

Santiago D. B., \& Gomes H. B. (2016) Estudo de Ilhas de Calor no Município de Maceió/AL, por meio de Dados Orbitais do Landsat 5. Revista Brasileira de Geografia Física, 9, 793-803.

Santiago, D. B., Gomes, H. B., \& Ferreira, L. S. (2019). Ilha de Calor e a Influência no Conforto Térmico da Região Integrada de Desenvolvimento da Grande Teresina (Ride). Rev. Bras. de Geo. Fis., 12 (1), 213-225.

Santos Y. S., Silva E. B., Oliveira-Júnior J. F., Santos P. J., \& Costa L. M. B. (2018). Diagnóstico da Morbidade e Mortalidade dos casos de Leptospirose no Nordeste Brasileiro entre 2000 a 2015. Enc. Biosfera, 15, 107-118.

Silva, J. S., da Silva, R. M., \& Santos, C. A. G. (2018). Spatiotemporal impact of land use/land cover changes on urban heat islands: A case study of Paço do Lumiar, Brazil. Building and Environment, 136, 279-292.

Silva, E. M. D. S., Correia, W. L. F., Oliveira, J. F. D., Barros, H. G., Costa, M. D. S., Gois, G. D., \& Falcão, N. A. D. M. (2021). Espaço-Temporalidade dos Focos de Calor na Região Metropolitana de Maceió. Revista Brasileira de Meteorologia, 35, 1029-1043.

Sousa, J. O., \& Martins Filho, J. (2019). Programa Minha Casa Minha Vida e os Impactos Socioambientais em Timon-MA: Uma Análise dos Conjuntos Habitacionais Padre Delfino e Julia Almeida. Rev. Equador, 8, 133-151. 
Research, Society and Development, v. 10, n. 11, e253101119537, 2021

(CC BY 4.0) | ISSN 2525-3409 | DOI: http://dx.doi.org/10.33448/rsd-v10i11.19537

USGS - United Stated Geological Survey. Using the USGS Landsat 8 Product, https://Landsat.usgs.gov/using-usgs-Landsat-8-product, 2018

Zeri M., Carvalho, V. S. B., Cunha-Zeri, G., Oliveira-Júnior, J. F., Lyra G. B., \& Freitas, E. D. (2016). Assessment of the variability of pollutants concentration over the metropolitan area of São Paulo, Brazil, using the wavelet transform. Atmospheric Science Letters, 17, 87-95. 